\title{
Methodology and modular tools for aspiration-led analysis of LP models
}

\author{
Marek Makowski \\ International Institute for Applied Systems Analysis \\ A-2361 Laxenburg, Austria. \\ Tel: +43-2236-807561. Fax: +43-2236-71313. \\ e-mail: marek@iiasa.ac.at
}

\begin{abstract}
The paper presents a Multiple Criteria Model Analysis (MCMA) based approach to the analysis of Linear Programming (LP) models. A brief overview of different approaches based on aspiration-led MCDA is followed by an overview of the implemented methodology. The corresponding approach to design and implementation of model-based decision support systems is illustrated by its application to the regional water quality management problem of the Nitra River Basin (Slovakia).
\end{abstract}

\section{Keywords}

Decision making support, multiple-criteria optimization, aspiration-reservation-led decision support.

\section{INTRODUCTION}

Decision making often requires analysis of large amounts of data and complex relations. In such cases, an analysis of a mathematical model can support rational decision making. Computerized tools designed and implemented for such purposes are called Decision Support Systems (DSS). A DSS, which is typically a problem specific tool, helps in the evaluation of consequences of given decisions and advises what decision would be the best for achieving a given set of goals. In a traditional optimization approach, only one goal is used as an optimized performance index and constraints are set for other goals. Such an approach requires from the user both deep knowledge of and experience in using various Operations Research methods (including model building, optimization techniques, sensitivity analysis). Specialists in other fields and Decision Makers (DM) typically can not meet such requirements. Therefore, multiple-criteria model analyses (MCMA) are being more widely used. The advantages of using MCMA are their ability to handle several goals. This clearly corresponds to the needs of decision support because most of real-life problems are multiobjective. The main advantage of proper implementation of MCDA is due the way it is used. Namely, it helps to analyze the problem rather than providing a single optimal solution. In typical situations, the specification of a consistent set of at- 
tainable goals is practically impossible. Therefore a DM interactively changes goals upon analysis of solutions obtained for previously specified goals expressed in terms of aspiration and reservation values for each criterion. Such an approach is called aspiration-led decision support (ALDS). It corresponds well to the natural way of analysis of a broad range of different types of problems. Proper implementation of ALDS requires both deep knowledge of the underlying methodologies and technical skills. However, use of ALDS based DSS is not only easy. It's main advantage is due to the fact that it allows for analysis of the problem by non-specialists in Operations Research who can change his/her preferences during the analysis process and can easily generate and compare solutions (or alternatives) that correspond to those different preferences. This paper presents an extension of the ALDS aimed at allowing a user flexible specification of preferences, optionally in terms of Fuzzy Sets.

\section{MULTIPLE CRITERIA OPTIMIZATION BASED DECISION SUPPORT}

Any model-based DSS relies on mathematical programming models that can adequately represent decision situations. This means that the model can be used for predicting and evaluating consequences of decisions, which is a basic function of simulation based DSSs. In optimization based DSSs the model is also used to compute decisions that would result in attaining specified goals. A specification of a model to be used within a DSS differs from a specification of a traditional model used for simulation or for single-criterion optimization due to the way the model is used. In traditional approaches a number of constraints are added to the core of the model in order to implicitly define not only feasible but also acceptable solutions. This used to be a must for batch oriented optimization approaches but it should be avoided for the specification of a model that is to be used as a part of a DSS. Hence, it is practical to divide specification and generation of the model into two parts and the corresponding stages:

- First, a core model is specified and generated. This model contains only a set of constraints that correspond to logical and physical relations between variables.

- Second, during an interactive procedure a DM specifies goals and preferences, including values of objectives that he/she wants to achieve and to avoid. Such a specification usually results in the generation of additional constraints and variables, which are added to the core model thus forming an optimization problem.

Such an approach has several advantages over the traditional approach in which both a preferential structure of a user and logical and physical relations between variables are specified and implemented together. Some of the advantages of the two-stage approach are listed below:

- A core model defines implicitly a set of feasible solutions. Feasibility is understood in the sense of logical and physical relations that must always hold. Therefore this part of a model (once the model is verified) should not be modified during analysis of the model. 
- A traditional model quite often has an unnecessarily narrow set of admissible solutions, which is caused by adding constraints aimed at making a solution not only feasible but also acceptable. Such additional constraints correspond to a preferential structure of a user and its implementation is done in a way similar to the constraints representing logical and physical relations. However, this often results in leaving out many interesting solutions beyond the analysis (because such solutions are not considered to be feasible in the strict sense of mathematical programming).

- Interactive analysis of the model is aimed at generation and analysis of rational solutions. Therefore a DM specifies interactively preferences that narrow the set of acceptable solutions. In other words, a DM examines solutions that fulfill both constraints specified by the core model and additional requirements specified by a DM. A DM typically changes those requirements substantially upon analysis of previously obtained solutions. Contrary to the constraints specified by a core model (which must not be violated) additional requirements are very often not attainable therefore they should not be represented as hard constraints.

- An interactive analysis of the model can be done with the help of modular tools that are not problem specific and can be used for a class of problems. Hence, software development is easier. Moreover, different methodologies and corresponding software modules for interactive analysis can be used without changing a core model formulation.

A more detailed discussion of specification of a core model and different traditional approaches to the model analysis for decision support is beyond the scope of this paper. A reader interested in those issues may want to consult (Makowski 1994b).

For the sake of brevity we have to skip the discussion of different multiple criteria based approaches. Those are discussed in detail e.g. by Gardiner and Steuer (1994) and by Makowski (1994b). Instead, we briefly summarize only the aspiration level based approach, originally proposed by Wierzbicki (1980). The essence of this method can be summarized as follows:

1. The DM selects, out of the potential objectives, a number (here denoted by $n$ ) of variables that will serve as criteria for evaluations of feasible solutions $x \in X_{0}$ defined by a core model. In typical applications there are $2-7$ criteria.

2. The DM specifies (with a help of an interactive tool) an aspiration level composed of the values that he/she wants to achieve for each criterion. Therefore, $\bar{q}=\left\{\bar{q}_{1}, \ldots, \bar{q}_{n}\right\}$.

3. The problem is transformed by a DSS into an auxiliary parametric single-objective problem. Its solution gives a Pareto-optimal point. If a specified aspiration level $\bar{q}$ is not attainable, then the Pareto-optimal point is the nearest (in the sense of a Chebyshev weighted norm) to the aspiration level. If the aspiration level is attainable, then the Pareto-optimal point is uniformly better than $\vec{q}$.

4. The DM explores various Pareto-optimal points by changing the aspiration levels $\bar{q}$. The underlying formulation of the problem is minimization of an achievement scalarizing function that can be interpreted as an ad-hoc non-stationary approximation of the DM's value function, depending on the currently selected aspiration level.

5. The procedures described in points 2,3 and 4 are repeated until a satisfactory solution is found. 
The selection of the Pareto-optimal point depends on the definition of the achievement scalarizing function, which includes also a selected aspiration point. Most of the methods use the achievement scalarizing function in the form:

$s(q, \bar{q}, w)=\max _{1 \leq i \leq n}\left\{w_{i}\left(q_{i}-\bar{q}_{i}\right)\right\}+\epsilon \sum_{i=1}^{n} w_{i}\left(q_{i}-\bar{q}_{i}\right)$

where $q(x) \in R^{n}$ is a vector of criteria, $\bar{q} \in R^{n}$ is an aspiration point, $w_{i}>0$ are scaling coefficients and $\epsilon$ is a given small positive number. Minimization of (1) for $x \in X_{0}$ generates a properly efficient solution with trade-off coefficients less then $(1+1 / \epsilon)$. Setting a value of $\epsilon$ is itself a trade-off between getting a too restricted set of properly Pareto solutions or a too wide set practically equivalent to weakly Pareto optimal solutions. Assuming the $\epsilon$ parameter to be of a technical nature, the selection of efficient solutions is controlled by the two vector parameters: $\bar{q}$ and $w$.

There is a common agreement that the aspiration point is a very good controlling parameter for examining a Pareto set. Much less attention is given to the problem of defining the weighting* coefficients $w$. A detailed discussion on weights in a scalarizing function is beyond the scope of this paper. The four commonly used approaches are summarized by Makowski (1994b). In practical applications the most promising approach is based on calculation of weights (that are used in definition of Chebyshev norm mentioned above) with help of the aspiration level $\bar{q}$ and a reservation level $q$ (the latter is composed of values of criteria that a user wants to avoid). Such approach has been introduced by the DIDAS family (described in (Lewandowski and Wierzbicki 1989)) of DSS.

\section{ASPIRATION/RESERVATION LED DECISION SUPPORT}

Following Ogryczak and Lahoda (1992) we will use for Aspiration-Reservation Based Decision Support techniques the acronym ARBDS. The ARBDS is an extension of the aspiration-based approach summarized in Section 2 and is based on the methodology proposed by Wierzbicki (1986), who also formulated general properties for the achievement scalarizing function. The achievement scalarizing function takes the form:

$\mathcal{S}(q, \bar{q}, \underline{q})=\min _{1 \leq i \leq n} u_{i}\left(q_{i}, \bar{q}_{i}, \underline{q}_{i}\right)+\epsilon \sum_{i=1}^{n} u_{i}\left(q_{i}, \bar{q}_{i}, \underline{q}_{i}\right)$

where $\bar{q}, \underline{q}$ are aspiration and reservation levels, respectively. Maximization of the function (2) over the set of feasible solutions defined by the corresponding core model provides a properly Pareto-optimal solution with the trade-off coefficient smaller than $(1+1 / \epsilon)$. Component achievement functions $u_{i}(\cdot)$ are strictly monotone (decreasing for minimized and increasing for maximized criteria, respectively) functions of the objective vector component $q_{i}$ with values

$u_{i}\left(q_{i}^{U}, \cdot\right)=1+\bar{\beta}, \quad u_{i}\left(\bar{q}_{i}, \cdot\right)=1, \quad u_{i}\left(\underline{q}_{i}, \cdot\right)=0, \quad u_{i}\left(q_{i}^{N}, \cdot\right)=-\bar{\eta}$

\footnotetext{
* Note that the weights $w$ should not (see e.g. (Makowski 1994b, Nakayama 1994)) be used for a conversion of a multiple criteria problem into a into a single criterion problem with a weighted sum of criteria.
} 
where $\bar{\beta}$ and $\bar{\eta}$, are given positive constants, typically equal to 0.1 and 10 , respectively.

The piece-wise linear component achievement functions $u_{i}$ proposed by Wierzbicki (1986) are defined by (4) and by (5) for minimized and maximized criteria, respectively.

$u_{i}(q, \bar{q}, \underline{q})=\left\{\begin{array}{lll}\alpha_{i} w_{i}\left(\bar{q}_{i}-q_{i}\right)+1, & \text { if } & q_{i}<\bar{q}_{i} \\ w_{i}\left(\bar{q}_{i}-q_{i}\right)+1, & \text { if } & \bar{q}_{i} \leq q_{i} \leq q_{i} \\ \beta_{i} w_{i}\left(\underline{q}_{i}-q_{i}\right) & \text { if } & \underline{q}_{i}<q_{i}\end{array}\right.$

$u_{i}(q, \bar{q}, \underline{q})=\left\{\begin{array}{lll}\alpha_{i} w_{i}\left(q_{i}-q_{i}\right) & \text { if } & q_{i}<q_{i} \\ w_{i}\left(\bar{q}_{i}-q_{i}\right)+1, & \text { if } & q_{i} \leq q_{i} \leq \bar{q}_{i} \\ \beta w_{i}\left(\bar{q}_{i}-q_{i}\right)+1, & \text { if } & \bar{q}_{i}<q_{i}\end{array}\right.$

where $w_{i}=1 /\left(\underline{q}_{i}-\bar{q}_{i}\right)$, and $\alpha_{i}, \beta_{i}(i=1,2, \ldots, n)$ are given parameters. The parameters $\alpha_{i}$ and $\beta_{i}$ are set in such a way that $u_{i}$ takes the values defined by (3).

The ARBDS method outlined above can be also interpreted in terms of fuzzy sets as an extension of interactive fuzzy multi-objective programming as proposed by Seo and Sakawa (1988). In this approach the membership function is not elicited at an initial iteration but the user is allowed to interactively change it upon analysis of obtained solutions. This approach assumes the classical form of the membership function originally proposed by Zadeh (1965). However, in order to properly handle - within the framework of the component achievement function - the criteria's values worse than a reservation level, and better than an aspiration level, it is necessary to allow for values of a membership function that are negative or greater than one. Such an extension of the membership function has been proposed by Granat and Wierzbicki (1994), who also suggested a method of constructing order-consistent component achievement scalarizing functions based on membership functions describing the satisfaction of the user with the attainment of separate objectives.

The modular tool called LP-Multi (Makowski 1994b) handles the multicriteria analysis of LP and MIP models. It allows a user specification, in addition to aspiration and reservation levels for each criterion, and also interactive specification of preferences for criteria values between aspiration and reservation levels. Therefore, the piece-wise linear functions $u_{i}(4,5)$ take a more general form. Namely they are defined by segments $u_{j i}$ :

$u_{j i}=\alpha_{j i} q_{i}+\beta_{j i}, \quad q_{j i} \leq q_{i} \leq q_{j+1, i} \quad j=1, \ldots, p_{i}$

where $p_{i}$ is a number of segments for $i$-th criterion,

$\alpha_{j i}=\frac{u_{j+1, i}-u_{j i}}{q_{j+1, i}-q_{j i}}$

$\beta_{j i}=u_{j i}-\alpha_{j i} q_{j i}$

where points $\left(u_{j i}, q_{i j}\right)$ are interactively defined with the help of FT-Tool due to Granat and Makowski (1995). Concavity of the piece-wise linear function $u(q)$ defined by (6) can be assured by a condition:

$\alpha_{1 i}>\alpha_{2 i}>\ldots>\alpha_{p_{i} i}$ 
This condition corresponds well to the nature of the problem since one accepts small changes of $u_{i}$ when a criterion value is better or close to an aspiration level. The speed of such change should increase along with moving towards a reservation level and should increase even faster between reservation and nadir points. Such features are consistent with the commonly known properties of the membership function used in applications based on the fuzzy set approach. Therefore such an interpretation of the extended-valued membership function makes it possible to combine the extensions of the two methods: Aspiration Reservation Based Multiple-Criteria Optimization and Fuzzy Multi-objective Linear Programming into a uniform approach described in more detail by Makowski (1994b).

\section{DESIGN AND IMPLEMENTATION}

Although a DSS must be problem specific, there are methodologies and tools applicable to many different problems. Such methodologies and reusable modular tools have been developed by the Methodology of Decision Analysis Project and have been applied, in collaboration with other projects at IIASA, to several problems. For the sake of illustration we outline only one application, namely, the regional water quality management problem of the Nitra River Basin (Slovakia) documented by Makowski, Somlyódy and Watkins (1995). We consider a river basin or a larger region composed of several basins where the water quality is extremely poor. We also consider a set of waste water treatment plants (either existing or to be possibly constructed) and, at each plant, some technology (which may be composed of a set of technologies to be selected out of a bigger given set of possible technologies) that can be implemented in order to improve the water quality in a region. The traditional optimization based approach to solving such a problem consists of looking for a set of plants and technologies whose implementation would result in maintaining prescribed water quality standards at minimum cost. However, the application of such an approach would in this case, as in many other cases, result in an infeasible solution because of the costs involved. Therefore another approach to decision support has been applied for the Nitra River Basin. Namely, a system of models has been developed for supporting a decision making process. The system is composed of simulation and single criterion dynamic programming models by (Somlyódy, Masliev, Petrovic and Kularathna 1994) and of an aspiration-led multiple criteria optimization model due to Makowski et al. (1995) and it is envisaged to serve two purposes. First, as a decision-aid tool for analysts and high-level decision makers in establishing the effluent and/or ambient water quality standards and the associated appropriate economic instruments that can be enforced to control the waste water discharges. Second, to aid in evaluation of alternative treatment strategies (technologies in treatment plants) and/or in selecting the most appropriate strategy based on the water quality standards and on the costs (capital investment and operational).

The aspiration-led component of this DSS is composed of the following modular and portable software tools (see Figure 1):

1. A Graphical User Interface (GUI) for handling all the interaction with the user. It is linked with FT-Tool (Granat and Makowski 1995) that allows for interactive specification of aspiration and reservation levels (and optionally piece-wise linear membership function for criteria values between those levels), as well as the changing of a criterion's 


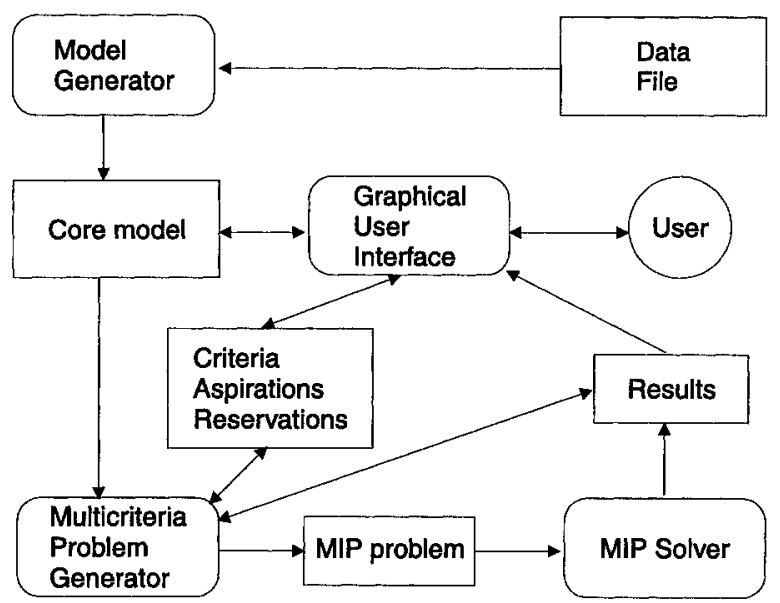

Figure 1 The structure of a Decision Support System for the water quality management in the Nitra River Basin.

status. Another modular tool, LP-Multi (see (Makowski 1994b) for details), processes the multiple criteria problem using the approach outlined in Section 3 . The resulting MIP problem is based on the core model and the aspiration and reservation levels which represent current preferential structure of a DM.

2. A problem-specific model generator for generating the core model which relates waste water emissions, treatment decisions, and the resulting ambient water quality. It is important that the core model include only physical and logical relations, and not the preferential structure of the DM.

3. A modular solver for mixed integer programming problems MOMIP developed by Ogryczak and Zorychta (1994).

4. A data interchange tool LP-DIT described by Makowski (1994a). This tool provides an easy and efficient way for the definition and modification of MIP problems, as well as the interchange of data between a problem generator, a solver, and software modules which serve for problem modification and solution analysis.

Portability of the developed tools is achieved by using $\mathrm{C}++$ programming language and a portable commercial tool for Graphical User Interface (GUI) documented in (Inm 1993b, Inm 1993a). Such an approach allows for reuse of most of the components needed for a DSS applied to other problems. It also facilitates experiments with different solvers and with modules providing problem specific interaction with a user. Note, that a new application only requires development of a model generator and, optionally, a problem specific module for a more detailed analysis of results. Hence, the approach presented is useful for fast development of DSS and for analysis of mathematical programming problems that result from the research activities at IIASA. 


\section{REFERENCES}

Gardiner, L. and Steuer, R. (1994). Unified interactive multiple objective programming, European Journal of Operational Research 74: 391-406.

Granat, J. and Makowski, M. (1995). FT - Modular tool for interactive specification of preferences in terms of fuzzy sets, Working Paper WP-95-73, International Institute for Applied Systems Analysis, Laxenburg, Austria.

Granat, J. and Wierzbicki, A. P. (1994). Interactive specification of DSS user preferences in terms of fuzzy sets, Working Paper WP-94-29, International Institute for Applied Systems Analysis, Laxenburg, Austria.

Inm (1993a). zApp Interface Pack, Programmer's Guide 83 Reference.

Inm (1993b). zApp, The Portable C++ Application Framework, Programmer's Guide.

Lewandowski, A. and Wierzbicki, A. (eds) (1989). Aspiration Based Decision Support Systems: Theory, Software and Applications, Vol. 331 of Lecture Notes in Economics and Mathematical Systems, Springer Verlag, Berlin, New York.

Makowski, M. (1994a). LP-DIT, Data Interchange Tool for Linear Programming Problems, (version 1.20), Working Paper WP-94-36, International Institute for Applied Systems Analysis, Laxenburg, Austria.

Makowski, M. (1994b). Methodology and a modular tool for multiple criteria analysis of LP models, Working Paper WP-94-102, International Institute for Applied Systems Analysis, Laxenburg, Austria.

Makowski, M., Somlyódy, L. and Watkins, D. (1995). Multiple criteria analysis for water quality management in the Nitra basin, Water Resources Bulletin. Submitted in May 1995.

Nakayama, H. (1994). Aspiration level approach to interactive multi-objective programming and its applications, Working Paper WP-94-112, International Institute for Applied Systems Analysis, Laxenburg, Austria.

Ogryczak, W. and Lahoda, S. (1992). Aspiration/reservation-based decision support - a step beyond goal programming, Journal of Multi-Criteria Decision Analysis 1(2): 101117.

Ogryczak, W. and Zorychta, K. (1994). Modular optimizer for mixed integer programming, MomIP version 2.1, Working Paper WP-94-35, International Institute for Applied Systems Analysis, Laxenburg, Austria.

Seo, F. and Sakawa, M. (1988). Multiple Criteria Decision Analysis in Regional Planning: Concepts, Methods and Applications, D. Reidel Publishing Company, Dordrecht.

Somlyódy, L., Masliev, I., Petrovic, P. and Kularathna, M. (1994). Water quality management in the Nitra River Basin, Collaborative Paper CP-94-02, International Institute for Applied Systems Analysis, Laxenburg, Austria.

Wierzbicki, A. (1980). The use of reference objectives in multiobjective optimization, in G. Fandel and T. Gal (eds), Multiple Criteria Decision Making, Theory and Applications, Vol. 177 of Lecture Notes in Economics and Mathematical Systems, Springer Verlag, Berlin, New York, pp. 468-486.

Wierzbicki, A. (1986). On the completeness and constructiveness of parametric characterizations to vector optimization problems; OR Spectrum 8: 73-87.

Zadeh, L. (1965). Fuzzy sets, Information and Control 8: 338-353. 